\title{
Cubic Transmuted Dagum Distribution: Properties and Applications
}

\author{
Adana'a Felix Chama \\ Department of Mathematical Sciences, Taraba State University, Jalingo, Nigeria \\ Email address: \\ chamaadanaa@gmail.com \\ To cite this article: \\ Adana'a Felix Chama. Cubic Transmuted Dagum Distribution: Properties and Applications. Mathematics Letters. \\ Vol. 7, No. 1, 2021, pp. 7-18. doi: 10.11648/j.ml.20210701.12
}

Received: November 26, 2020; Accepted: December 10, 2020; Published: April 30, 2021

\begin{abstract}
In this article, a generalization of the new cubic transmuted Dagum (CTD) distribution is derived and have developed a cubic transmuted family of distribution. The proposed distribution includes a special cases of the new Transmuted Dagum distribution (TDD). The objectives are to model a new distribution called cubic transmuted Dagum distribution to take care of the flexibility and multimodal (complex) effect which the transmuted form of the distribution cannot handle. The work comprises of the probability density function of cubic transmuted Dagum distribution and its Cumulative distribution function and attempt was made to compare the new distribution with the transmuted form of the distribution. Various structural properties of the new distribution, including the moments, characteristic function, quantile, moment generating function, mean, variance, reliability analysis, order statistics are derived. The maximum likelihood estimation method has been proposed for the estimation of the parameters of the Cubic transmuted Dagum distribution. The usefulness of the derived model is illustrated using two data sets to compare the performance of the new distribution with the transmuted form of the distribution and also with the parent (Dagum) distribution, and it is proved that CTD distribution is a better distribution than the transmuted Dagum distribution and the Dagum distributions based on some goodness of fit measures. Therefore, we conclude that the new model fits real life data better than the transmuted form and the base distribution. Also cubic transmuted Dagum distribution attracts more applications in several areas such as engineering, survival data, economics and others.
\end{abstract}

Keywords: Transmuted Distribution, Dagum Distribution, Reliability Function, Moment Generating Function, Maximum Likelihood Estimation

\section{Introduction}

Dagum distribution is widely used for modeling a wide range of data in several fields. It is very worthwhile for analyzing income distribution, actuarial, metrological data and equally preferable for survival analysis [6, 1]. Moreover, it is considered to be the most suitable choice as compared to other three parameter distributions in several cases [2, 3]. It belongs to the generalized Beta distribution and is generated from generalized Beta-II by considering a shape parameter one and referred as inverse Burr distribution. The independent derivation of the Dagum distribution was done [7]. While Bordley et al studied the income and income related data by Dagum distributions [8]. An attempt to fit this distribution on family incomes data for the United States and proved that its performance is the best among all the models was performed [6]. Also Bordley studied the United States family income data by probability distributions along with the Dagum distribution [8]. In a study [9] revealed after the study of 23 countries' income data, the Dagum distribution is the best among the two and three parameter distributions. Also, Alwan et al tried more than fifty distributions to model the reliability of the electrical distribution system and finally the Dagum distribution was considered as the best choice [10]. We have cited very few studies but various other related studies also confirm the better performance of the Dagum distribution. Recently [6, 4] transmuted the Dagum distribution to broadened its application to lifetime data found that it is more appropriate than the parent distribution 
and more flexible to life time changes to different shapes of the distribution. In this study, is a proposal for a the cubic transmuted Dagum distribution to model survival data to take care of the flexibility of the data and complexity (multimodal) in survival data

\subsection{Importance of the Study}

The study will so much contribute to the knowledge of the uses of Dagum distribution in Biostatistics, Engineering, History, Economics, sociology for analyzing and modeling of more complex (multi-modal) reliability study, events history analysis, policy duration analysis etc.

\subsection{Aim and Objectives of the Study}

The aim of the study is to model a cubic transmuted Dagum distribution to real life data, and the objectives are to:

Determine a new model called cubic transmuted Dagum distribution.

Determine the properties of cubic transmuted Dagum distribution.

Compare the cubic transmuted Dagum distribution with Dagum distribution and transmuted Dagum distribution.

Make recommendations based on the findings.

\subsection{Scope and Limitation}

The study will focus on the application of the cubic transmuted Dagum distribution to model the survival function of a distribution and the hazard function. The mathematical derivations of some expressions such as the mean, variance, moment generating function, characteristic function, probability density function, and order statistics will also be made.

$$
\begin{gathered}
F_{C T D D}(x)=(1-\lambda)\left[\left(1+\alpha x^{-\theta}\right)^{-\beta}\right]+3 \lambda\left[\left(1+\alpha x^{-\theta}\right)^{-\beta}\right]^{2}-2 \lambda\left[\left(1+\alpha x^{-\theta}\right)^{-\beta}\right]^{3} \\
F_{C T D D}(x)=(1-\lambda)\left(1+\alpha x^{-\theta}\right)^{-\beta}+3 \lambda\left(1+\alpha x^{-\theta}\right)^{-2 \beta}-2 \lambda\left(1+\alpha x^{-\theta}\right)^{-3 \beta}
\end{gathered}
$$

And its respective pdf of cubic transmuted Dagum distribution is given by substituting (3) and (4) into (2)

$$
\begin{gathered}
f_{C T D D}(x)=\alpha \theta \beta x^{-\theta-1}\left\{\left(1+\alpha x^{-\theta}\right)^{-\beta-1}\left[\begin{array}{c}
1-\lambda \\
+6 \lambda
\end{array}\right]\left(1+\alpha x^{-\theta}\right)^{-\beta}-6 \lambda\left[\left(1+\alpha x^{-\theta}\right)^{-\beta}\right]^{2}\right\} \\
f_{C T D D}(x)=\alpha \theta \beta x^{-\theta-1}\left\{\begin{array}{c}
\left(1+\alpha x^{-\theta}\right)^{-\beta-1}[1-\lambda+6 \lambda] \\
\left(1+\alpha x^{-\theta}\right)^{-\beta}-6 \lambda\left[\left(1+\alpha x^{-\theta}\right)^{-2 \beta}\right]
\end{array}\right\}
\end{gathered}
$$

Where $x>0$, and $\alpha, \theta, \beta>0-1 \leq \lambda \leq-1$, and $\alpha$ is a scale parameter while $\theta$ and $\beta$ are the shape parametres and $\lambda$ is the cubic transmuted parameter of the cubic transmuted Dagum distribution. $f_{C T D D}(x)$ is the probability density function of the cubic transmuted Dagum distribution and $F_{C T D D}(x)$ is the cumulative distribution function of the cubic transmuted Dagum distribution. By varying the values $\lambda$ of the distribution due to cubic transmutation becomes more flexible robust to complex (multimodal) problems, hence (5) and (6) are the cumulative distribution function and probability function of the cubic transmuted Dagum distribution. The shapes of this density and cumulative distribution function assuming various combinations of parameters are illustrated in figure 1 below. 


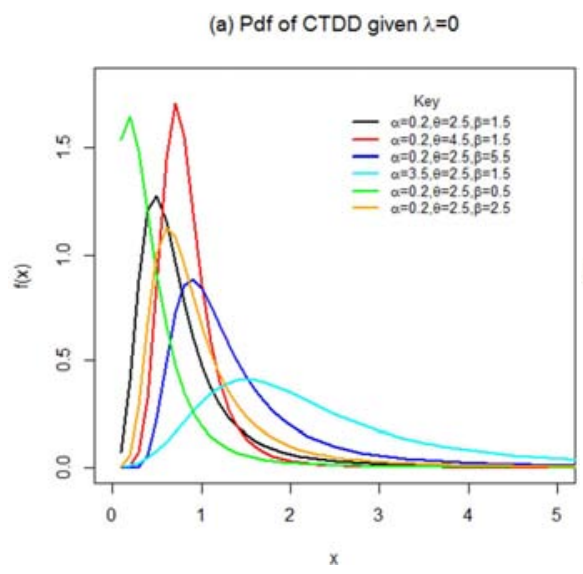

(d) Pdf of CTDD given $\alpha=0.2, \theta=2.5 \& \beta=5.5$

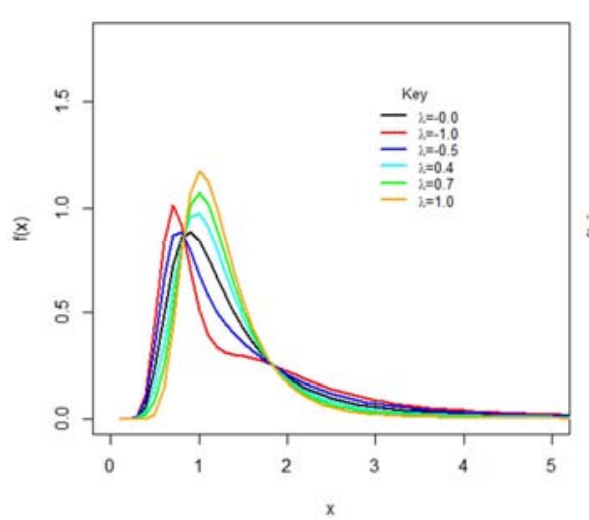

(b) Pdf of CTDD given $\alpha=0.2, \theta=2.5 \& \beta=1.5$

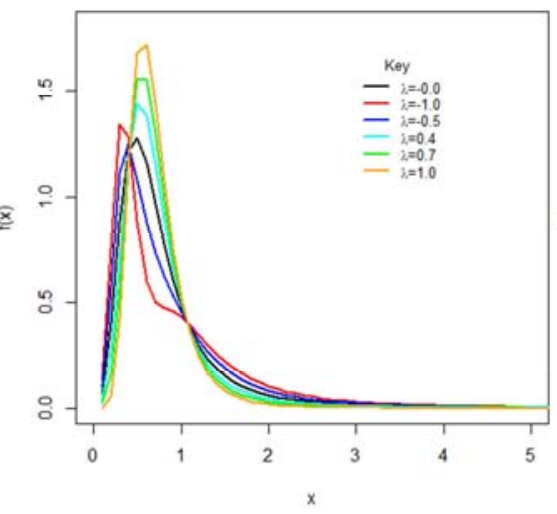

(e) Pdf of CTDD given $\alpha=3.5, \theta=2.5 \& \beta=1.5$

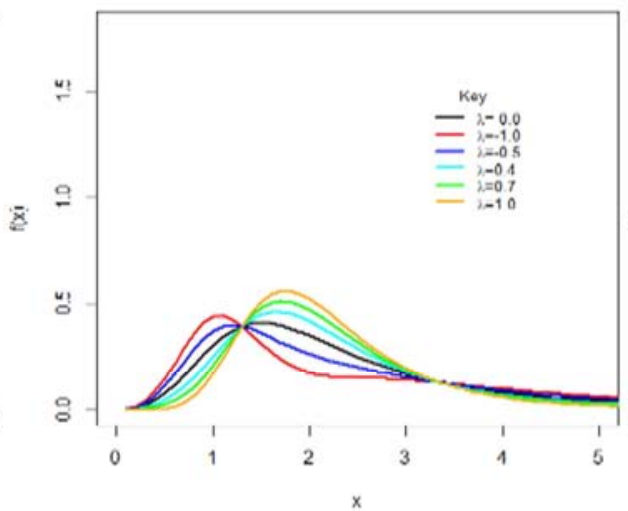

(c) Pdf of CTDD given $\alpha=0.2, \theta=4.5 \& \beta=1.5$

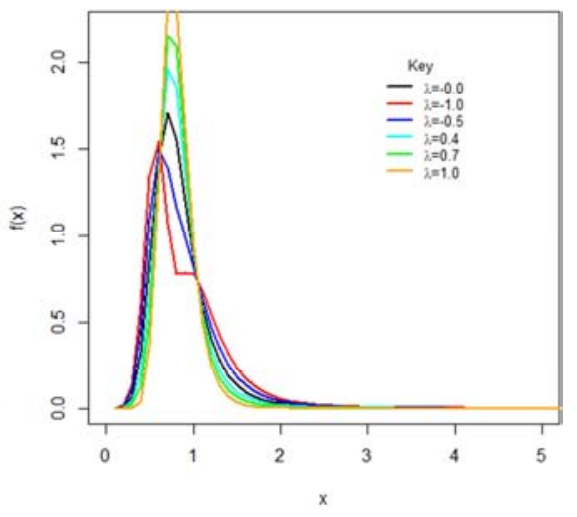

(f) Pdf of CTDD given $\alpha=0.2, \theta=2.5 \& \beta=2.5$

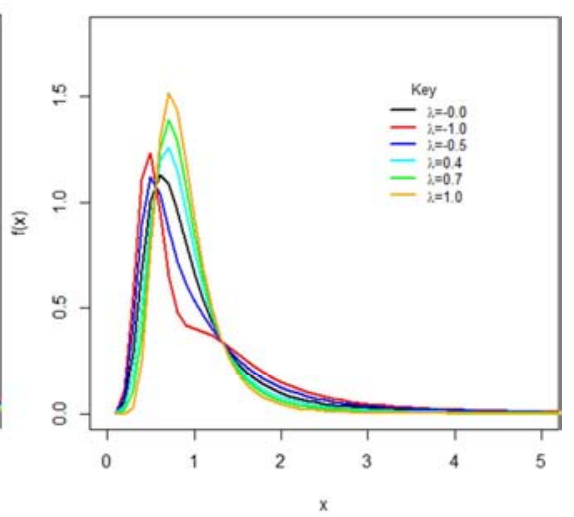

Figure 1. PDF of the CTDD for different values of the parameters.

(a) Cdf of CTDD given $\lambda=0$

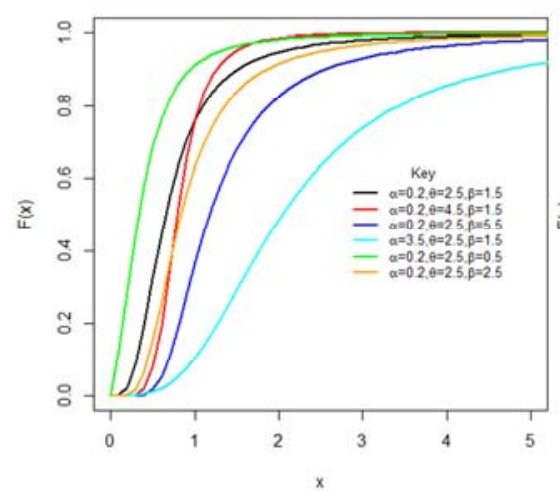

(d) Cdf of CTDD given $\alpha=0.2, \theta=2.5 \& \beta=5.5$

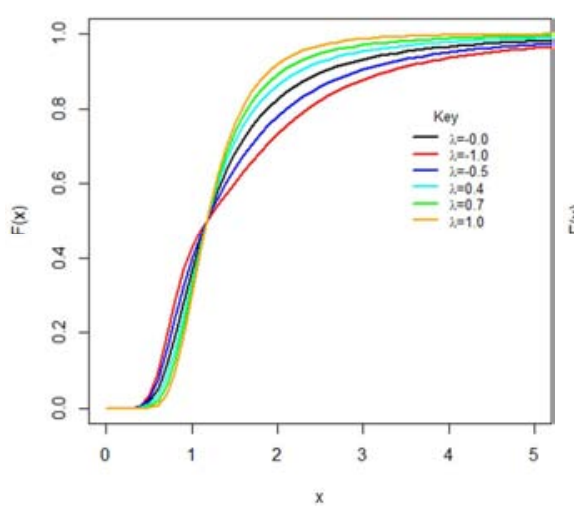

(b) Cdf of CTDD given $\alpha=0.2, \theta=2.5 \& \beta=1.5$

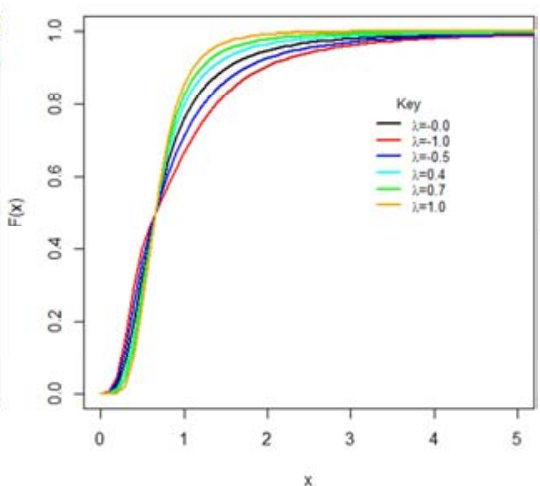

(e) Cdf of CTDD given $\alpha=3.5, \theta=2.5 \& \beta=1.5$

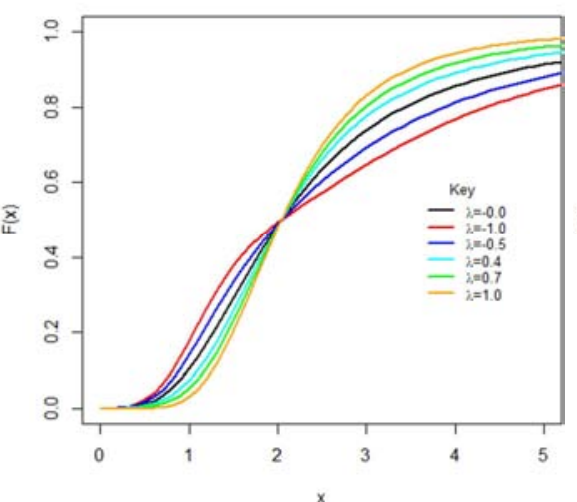

(c) Cdf of CTDD given $\alpha=0.2, \theta=4.5 \& \beta=1.5$

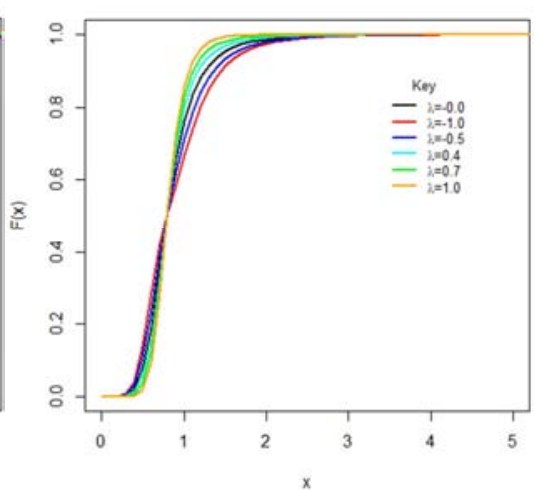

(f) Cdf of CTDD given $\alpha=0.2, \theta=2.5 \& \beta=2.5$

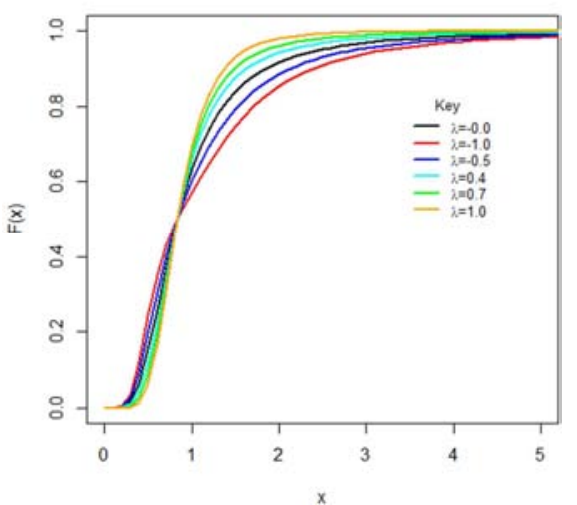

Figure 2. CDF of the CTDD for different values of the parameters. 


\subsection{Some Properties of the Proposed Cubic Transmuted Dagum Distribution}

Main statistical properties such as $\mathrm{r}^{\text {th }}$ moments, mean, variance, and moment Generating function for the transmuted Dagum distribution will be derived.

\subsubsection{Moments}

Moments are necessary and important in any statistical

$$
\mu_{n}^{\prime}=(1+\lambda) \beta \alpha^{\frac{n}{\theta}} B\left(1-\frac{n}{\theta}, \beta+\frac{n}{\theta}\right)+6 \lambda \beta \alpha^{\frac{n}{\theta}} B\left(1-\frac{n}{\theta}, 2 \beta+\frac{n}{\theta}\right)-6 \lambda \beta \alpha^{\frac{n}{\theta}} B
$$

proof:

$$
\mu_{n}^{\prime}=E\left[x^{n}\right]=\int_{0}^{\infty} x^{n} f(x) d x
$$

Where $f(x)$ is the pdf of the cubic transmuted Dagum distribution.

Recall. that,

$$
\begin{gathered}
f_{C T D D}(x)=\alpha \theta \beta x^{-\theta-1}\left\{\left(1+\alpha x^{-\theta}\right)^{-\beta-1}[1-\lambda+6 \lambda]\left(1+\alpha x^{-\theta}\right)^{-\beta}-6 \lambda\left[\left(1+\alpha x^{-\theta}\right)^{-2 \beta}\right]\right\} \\
f_{C T D D}(x)=(1-\lambda) \alpha \theta \beta x^{-\theta-1}\left(1+\alpha x^{-\theta}\right)^{-\beta-1}+6 \alpha \theta \beta \lambda x^{-\theta-1}\left(1+\alpha x^{-\theta}\right)^{-2 \beta-1}-6 \alpha \theta \beta \lambda x^{-\theta-1}\left(1+\alpha x^{-\theta}\right)^{-3 \beta-1}
\end{gathered}
$$

Then,

$$
\begin{aligned}
& \mu_{n}^{\prime}=E\left[x^{n}\right]=\int_{0}^{\infty} x^{n}\left[(1-\lambda) \alpha \theta \beta x^{-\theta-1}\left(1+\alpha x^{-\theta}\right)^{-\beta-1}+6 \alpha \theta \beta \lambda x^{-\theta-1}\left(1+\alpha x^{-\theta}\right)^{-2 \beta-1}-6 \alpha \theta \beta \lambda x^{-\theta-1}\left(1+\alpha x^{-\theta}\right)^{-3 \beta-1}\right] d x \\
& \mu_{n}^{\prime}=E\left[x^{n}\right]=(1-\lambda) \int_{0}^{\infty}\left[\alpha \theta \beta x^{n-\theta-1}\left(1+\alpha x^{-\theta}\right)^{-\beta-1}+6 \alpha \theta \beta \lambda x^{n-\theta-1}\left(1+\alpha x^{-\theta}\right)^{-2 \beta-1}-6 \alpha \theta \beta \lambda x^{n-\theta-1}\left(1+\alpha x^{-\theta}\right)^{-3 \beta-1}\right] d x
\end{aligned}
$$

But from transmuted Dagum distribution by [4]

$$
E\left[x^{n}\right]=\int_{0}^{\infty} x^{n} f_{D D}(x) d x=\int_{0}^{\infty} \alpha \theta \beta x^{-\theta-1}\left(1+\alpha x^{-\theta}\right)^{-\beta-1} d x=\beta \alpha^{\frac{n}{\theta}} B\left(1-\frac{n}{\theta}, \beta+\frac{n}{\theta}\right)
$$

Where $\mathrm{B}(\ldots)$ is the beta function defined by

$$
B(x, y)=\int_{0}^{1} e^{x-1}(1-t)^{y-1} d t
$$

Therefore

$\mu_{n}^{\prime}=(1+\lambda) \beta \alpha^{\frac{n}{\theta}} B\left(1-\frac{n}{\theta}, \beta+\frac{n}{\theta}\right)+6 \lambda \beta \alpha^{\frac{n}{\theta}} B\left(1-\frac{n}{\theta}, 2 \beta+\frac{n}{\theta}\right)-6 \lambda \beta \alpha^{\frac{n}{\theta}} B\left(1-\frac{n}{\theta}, 3 \beta+\frac{n}{\theta}\right)$ Hence the proof.

\subsubsection{The Mean}

The mean of the cubic transmuted Dagum distribution can be obtained from the $n^{\text {th }}$ moment of the distribution when $\mathrm{n}=1$, and is obtained as:

$$
\mu_{1}^{\prime}=(1+\lambda) \beta \alpha^{\frac{1}{\theta}} B\left(1-\frac{1}{\theta}, \beta+\frac{1}{\theta}\right)+6 \lambda \beta \alpha^{\frac{1}{\theta}} B\left(1-\frac{1}{\theta}, 2 \beta+\frac{1}{\theta}\right)-6 \lambda \beta \alpha^{\frac{1}{\theta}} B\left(1-\frac{1}{\theta}, 3 \beta+\frac{1}{\theta}\right)
$$

\subsubsection{The Variance}

The $n^{\text {th }}$ central moment or moment about the mean of $x$, can be obtained as:

$$
\mu_{n}=E\left[x-\mu_{1}^{\prime}\right]^{n}=\sum_{i=0}^{n}(-1)^{i}\left(\begin{array}{c}
n \\
i
\end{array}\right) \mu_{1}^{i} \mu_{n-1}
$$

Then the variance of the cubic transmuted Dagum distribution can be obtained from the $n^{\text {th }}$ central moment or moment about the mean of $x$ when $\mathrm{n}=2$.

$$
\mu_{2}^{\prime}=E\left(x^{2}\right)=(1+\lambda) \beta \alpha^{\frac{2}{\theta}} B\left(1-\frac{2}{\theta}, \beta+\frac{2}{\theta}\right)+6 \lambda \beta \alpha^{\frac{2}{\theta}} B\left(1-\frac{2}{\theta}, 2 \beta+\frac{2}{\theta}\right)-6 \lambda \beta \alpha^{\frac{2}{\theta}} B\left(1-\frac{2}{\theta}, 3 \beta+\frac{2}{\theta}\right)
$$




$$
\begin{gathered}
\operatorname{Var}(x)=E\left(x^{2}\right)-[E(x)]^{2} \\
\operatorname{Var}(x)=(1+\lambda) \beta \alpha^{\frac{2}{\theta}} B\left(1-\frac{2}{\theta}, \beta+\frac{2}{\theta}\right)+6 \lambda \beta \alpha^{\frac{2}{\theta}} B\left(1-\frac{2}{\theta}, 2 \beta+\frac{2}{\theta}\right)-6 \lambda \beta \alpha^{\frac{2}{\theta}} B\left(1-\frac{2}{\theta}, 3 \beta+\frac{2}{\theta}\right) \\
-\left\{(1+\lambda) \beta \alpha^{\frac{1}{\theta}} B\left(1-\frac{1}{\theta}, \beta+\frac{1}{\theta}\right)+6 \lambda \beta \alpha^{\frac{1}{\theta}} B\left(1-\frac{1}{\theta}, 2 \beta+\frac{1}{\theta}\right)-6 \lambda \beta \alpha^{\frac{1}{\theta}} B\left(1-\frac{1}{\theta}, 3 \beta+\frac{1}{\theta}\right)\right\}^{2} \\
\operatorname{Var}(x)=(1+\lambda) \beta \alpha^{\frac{2}{\theta}} B\left(1-\frac{2}{\theta}, \beta+\frac{2}{\theta}\right)+6 \lambda \beta \alpha^{\frac{2}{\theta}}\left\{B\left(1-\frac{2}{\theta}, 2 \beta+\frac{2}{\theta}\right)-B\left(1-\frac{2}{\theta}, 3 \beta+\frac{2}{\theta}\right)\right\}-\beta \alpha^{\frac{1}{\theta}}\left\{( 1 + \lambda ) B \left(1-\frac{1}{\theta}, \beta+\right.\right. \\
\left.\left.\frac{1}{\theta}\right)+6 \lambda B\left(1-\frac{1}{\theta}, 2 \beta+\frac{1}{\theta}\right)-6 \lambda B\left(1-\frac{1}{\theta}, 3 \beta+\frac{1}{\theta}\right)\right\}^{2}
\end{gathered}
$$

The following expression can be used to obtain the moment ratios for cubic transmuted Dagum distribution such as Coefficient of variation (CV), Skewness (Sk), Kurtosis (Kr) using $m_{r}^{\prime}(r=1,2,3,4)$.

$$
\begin{gathered}
C V=\frac{\delta}{m_{1}^{\prime}} \\
S k=\frac{m_{3}^{\prime}-2 m_{2}^{\prime} m_{1}^{\prime}+2\left(m_{1}^{\prime}\right)^{3}}{\delta^{2}} \\
K r=\frac{m_{4}^{\prime}-4 m_{3}^{\prime} m_{1}^{\prime}+6 m_{2}^{\prime}\left(m_{1}^{\prime}\right)^{2}-3\left(m_{1}^{\prime}\right)^{4}}{\delta^{4}}
\end{gathered}
$$

\subsubsection{Moment Generating Function}

This is a way of organizing all the moments into one

$$
M_{x}(t)=\sum_{k=0}^{\infty} \frac{t^{k}}{k !}\left\{(1-\lambda) \beta \alpha^{\frac{k}{\theta}} B\left(1-\frac{k}{\theta}, \beta+\frac{k}{\theta}\right)+6 \lambda \beta \alpha^{\frac{k}{\bar{\theta}}} B\left(1-\frac{k}{\theta}, 2 \beta+\frac{k}{\theta}\right)-6 \lambda \beta \alpha^{\frac{k}{\bar{\theta}}} B\left(1-\frac{k}{\theta}, 3 \beta+\frac{k}{\theta}\right)\right\}
$$

Proof: let the moment generating function be given as

$$
M_{x}(t)=E\left(e^{t x}\right)=\int_{0}^{\infty} e^{t x} f(x) d x
$$

Using the power series expansion [11].

$$
\begin{gathered}
e^{t x}=\sum_{k=0}^{\infty} \frac{(t x)^{k}}{k !}=\sum_{k=0}^{\infty} \frac{t^{k}}{k !} x^{k} \\
M_{x}(t)=E\left(e^{t x}\right)=\int_{0}^{\infty} \sum_{k=0}^{\infty} \frac{t^{k}}{k !} x^{k} f(x) d x \\
M_{x}(t)=E\left(e^{t x}\right)=\sum_{k=0}^{\infty} \frac{t^{k}}{k !} \int_{0}^{\infty} x^{k} f(x) d x
\end{gathered}
$$

But

$$
\mu_{k}=\int_{0}^{\infty} x^{k} f(x) d x
$$

Therefore

$$
M_{x}(t)=E\left(e^{t x}\right)=\sum_{k=0}^{\infty} \frac{t^{k}}{k !}\left\{(1-\lambda) \beta \alpha^{\frac{k}{\theta}} B\left(1-\frac{k}{\theta}, \beta+\frac{k}{\theta}\right)+6 \lambda \beta \alpha^{\frac{k}{\theta}} B\left(1-\frac{k}{\theta}, 2 \beta+\frac{k}{\theta}\right)-6 \lambda \beta \alpha^{\frac{k}{\theta}} B\left(1-\frac{k}{\theta}, 3 \beta+\frac{k}{\theta}\right)\right\}
$$

Hence the proof. 


\subsubsection{Characteristic Function}

The characteristic function has many useful and important properties which gives it a central role in statistical theory. Its approach is particularly useful for generating moments, characterization of distributions and in analysis of linear combination of independent random variables. A representation for the characteristics function is given by:

$$
\varphi_{x}(t)=E\left(e^{i t x}\right)=\int_{0}^{\infty} e^{i t x} f(x) d x
$$

Theorem 3: the characteristic function of a cubic transmuted Dagum distribution is given as

$$
\varphi_{x}(t)=E\left(e^{i t x}\right)=\sum_{k=0}^{\infty} \frac{(i t)^{k}}{k !}\left\{(1-\lambda) \beta \alpha^{\frac{k}{\theta}} B\left(1-\frac{k}{\theta}, \beta+\frac{k}{\theta}\right)+6 \lambda \beta \alpha^{\frac{k}{\theta}} B\left(1-\frac{k}{\theta}, 2 \beta+\frac{k}{\theta}\right)-6 \lambda \beta \alpha^{\frac{k}{\theta}} B\left(1-\frac{k}{\theta}, 3 \beta+\frac{k}{\theta}\right)\right\}
$$

Proof: let

$$
\varphi_{x}(\boldsymbol{t})=E\left(e^{i t x}\right)=\int_{0}^{\infty} e^{i t x} f(x) d x
$$

Using the power series expansion by[19]

$$
\begin{gathered}
e^{i t x}=\sum_{k=0}^{\infty} \frac{(i t x)^{k}}{k !}=\sum_{k=0}^{\infty} \frac{(i t)^{k}}{k !} x^{k} \\
\varphi_{x}(t)=E\left(e^{t x}\right)=\int_{0}^{\infty} \sum_{k=0}^{\infty} \frac{(i t)^{k}}{k !} x^{k} f(x) d x \\
\varphi_{x}(t)=E\left(e^{i t x}\right)=\sum_{k=0}^{\infty} \frac{(i t)^{k}}{k !} \int_{0}^{\infty} x^{k} f(x) d x
\end{gathered}
$$

But

$$
\mu_{k}=\int_{0}^{\infty} x^{k} f(x) d x
$$

Therefore

$$
\varphi_{x}(t)=E\left(e^{i t x}\right)=\sum_{k=0}^{\infty} \frac{(i t)^{k}}{k !}\left\{(1-\lambda) \beta \alpha^{\frac{k}{\theta}} B\left(1-\frac{k}{\theta}, \beta+\frac{k}{\theta}\right)+6 \lambda \beta \alpha^{\frac{k}{\theta}} B\left(1-\frac{k}{\theta}, 2 \beta+\frac{k}{\theta}\right)-6 \lambda \beta \alpha^{\frac{k}{\theta}} B\left(1-\frac{k}{\theta}, 3 \beta+\frac{k}{\theta}\right)\right\}
$$

Hence the proof.

\subsubsection{Quantile Function}

Quantile function is considered as an important quantity in each distribution and are used widely for simulation of that distribution and finding key percentiles. One can obtain the quantile function of a cubic transmuted Dagum distribution by using [12] method which defined the quantile function as in the form $Q(u)=F^{-1}(u)$ where $Q(u)$ is define as the quantile function of $F(x)$ for $0<u<1$.

Theorem4: the quantile function $Q(u)$ of a cubic transmuted Dagum distribution is given as:

$$
Q(u)=\left\{\frac{1}{\alpha}\left[\left(\sqrt[3]{\left(\frac{1}{8}+\frac{3(1-\lambda)}{24 \lambda}-\frac{u}{4 \lambda}\right)+\sqrt{\left(\frac{1}{8}+\frac{3(1-\lambda)}{24 \lambda}-\frac{u}{4 \lambda}\right)^{2}+\left(\frac{(1-\lambda)}{-6 \lambda}-\frac{1}{4}\right)^{3}}}+\sqrt[3]{\left.\left.\left(\frac{1}{8}+\frac{3(1-\lambda)}{24 \lambda}-\frac{u}{4 \lambda}\right)-\sqrt{\left(\frac{1}{8}+\frac{3(1-\lambda)}{24 \lambda}-\frac{u}{4 \lambda}\right)^{2}+\left(\frac{(1-\lambda)}{-6 \lambda}-\frac{1}{4}\right)^{3}}+\frac{1}{2}\right)^{\frac{1}{\beta}}-1\right]}\right]\right\}^{\frac{1}{\theta}}\right.
$$

Proof: let $F(x)$ be the cumulative distribution function of the cubic transmuted Dagum distribution, and taking its inverse will give us the quantile function

Quantile function divides the ordered data into q equal sized portions. The smallest and largest value of the ordered data corresponds to probability 0 and 1 , respectively. The $q^{\text {th }}$ quantile of cubic transmuted Dagum distribution is obtained using 3.5 


$$
\begin{gathered}
F(x)=(1-\lambda)\left(1+\alpha x^{-\theta}\right)^{-\beta}+3 \lambda\left(1+\alpha x^{-\theta}\right)^{-2 \beta}-2 \lambda\left(1+\alpha x^{-\theta}\right)^{-3 \beta}=u \\
(1-\lambda)\left(1+\alpha x^{-\theta}\right)^{-\beta}+3 \lambda\left(1+\alpha x^{-\theta}\right)^{-2 \beta}-2 \lambda\left(1+\alpha x^{-\theta}\right)^{-3 \beta}-u=0
\end{gathered}
$$

Equation (3.14) is in form of polynomial of third order given as

$$
a m^{3}+b m^{2}+c m+d=0
$$

Letting

$$
m=\left(1+\alpha x^{-\theta}\right)^{-\beta}, a=-2 \lambda, b=3 \lambda, c=(1-\lambda), d=-u
$$

Therefore, equation (3.150 is of order three which can be solved using Cardano's formula in [13], which is given as follows:

$$
m=\sqrt[3]{\left(\frac{-b^{3}}{27 a^{3}}+\frac{b c}{6 a^{2}}-\frac{d}{2 a}\right)+\sqrt{\left(\frac{-b^{3}}{27 a^{3}}+\frac{b c}{6 a^{2}}-\frac{d}{2 a}\right)^{2}+\left(\frac{c}{3 a}-\frac{b^{2}}{9 a^{2}}\right)^{3}}}+\sqrt[3]{\left(\frac{-b^{3}}{27 a^{3}}+\frac{b c}{6 a^{2}}-\frac{d}{2 a}\right)-\sqrt{\left(\frac{-b^{3}}{27 a^{3}}+\frac{b c}{6 a^{2}}-\frac{d}{2 a}\right)^{2}+\left(\frac{c}{3 a}-\frac{b^{2}}{9 a^{2}}\right)^{3}}}-\frac{b}{3 a}
$$

Substituting m,a,b,c, and d in to (16)

$$
\left(1+\alpha x^{-\theta}\right)^{-\beta}=\sqrt[3]{\left(\frac{1}{8}+\frac{3(1-\lambda)}{24 \lambda}-\frac{u}{4 \lambda}\right)+\sqrt{\left(\frac{1}{8}+\frac{3(1-\lambda)}{24 \lambda}-\frac{u}{4 \lambda}\right)^{2}+\left(\frac{(1-\lambda)}{-6 \lambda}-\frac{1}{4}\right)^{3}}}+\sqrt[3]{\left(\frac{1}{8}+\frac{3(1-\lambda)}{24 \lambda}-\frac{u}{4 \lambda}\right)-\sqrt{\left(\frac{1}{8}+\frac{3(1-\lambda)}{24 \lambda}-\frac{u}{4 \lambda}\right)^{2}+\left(\frac{(1-\lambda)}{-6 \lambda}-\frac{1}{4}\right)^{3}}+\frac{1}{2}}
$$

Multiply the index of both side with $-\frac{1}{\beta}$

$$
1+\alpha x^{-\theta}=\left\{\sqrt[3]{\left(\frac{1}{8}+\frac{3(1-\lambda)}{24 \lambda}-\frac{u}{4 \lambda}\right)+\sqrt{\left(\frac{1}{8}+\frac{3(1-\lambda)}{24 \lambda}-\frac{u}{4 \lambda}\right)^{2}+\left(\frac{(1-\lambda)}{-6 \lambda}-\frac{1}{4}\right)^{3}}}+\sqrt[3]{\left.\left(\frac{1}{8}+\frac{3(1-\lambda)}{24 \lambda}-\frac{u}{4 \lambda}\right)-\sqrt{\left(\frac{1}{8}+\frac{3(1-\lambda)}{24 \lambda}-\frac{u}{4 \lambda}\right)^{2}+\left(\frac{(1-\lambda)}{-6 \lambda}-\frac{1}{4}\right)^{3}}+\frac{1}{2}\right\}^{-\frac{1}{\beta}}}\right.
$$

Subtract 1 from both side

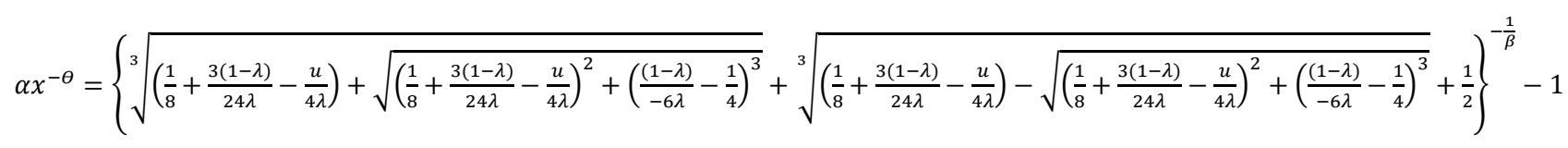

Divide both side by $\alpha$

$$
x^{-\theta}=\left[\frac { 1 } { \alpha } \left\{\sqrt[3]{\left(\frac{1}{8}+\frac{3(1-\lambda)}{24 \lambda}-\frac{u}{4 \lambda}\right)+\sqrt{\left(\frac{1}{8}+\frac{3(1-\lambda)}{24 \lambda}-\frac{u}{4 \lambda}\right)^{2}+\left(\frac{(1-\lambda)}{-6 \lambda}-\frac{1}{4}\right)^{3}}}+\sqrt[3]{\left.\left.\left(\frac{1}{8}+\frac{3(1-\lambda)}{24 \lambda}-\frac{u}{4 \lambda}\right)-\sqrt{\left(\frac{1}{8}+\frac{3(1-\lambda)}{24 \lambda}-\frac{u}{4 \lambda}\right)^{2}+\left(\frac{(1-\lambda)}{-6 \lambda}-\frac{1}{4}\right)^{3}}+\frac{1}{2}\right\}^{-\frac{1}{\beta}}-1\right]}\right.\right.
$$

Multiply the index of both side with $-\frac{1}{\theta}$

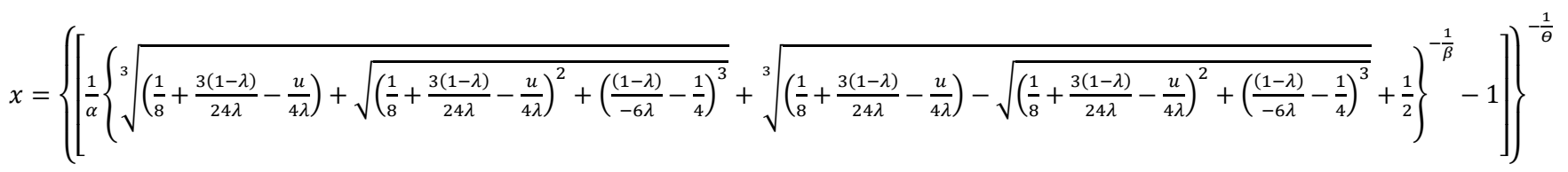

Therefore,

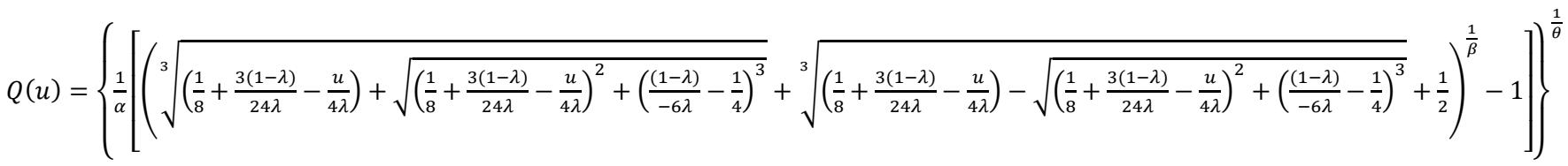

Hence the proof

\subsubsection{Survival Function}

Survival function is the probability function that a system or an individual will survive beyond a given time. Mathematically, 
survival function is given by:

$S(x)=1-F(x)$ where $F(x)$ is the cdf of the proposed cubic transmuted distribution in (3.6) above and is given as:

$$
\begin{gathered}
S(x)=1-\left\{(1-\lambda)\left(1+\alpha x^{-\theta}\right)^{-\beta}+3 \lambda\left[\left(1+\alpha x^{-\theta}\right)^{-\beta}\right]^{2}-2 \lambda\left[\left(1+\alpha x^{-\theta}\right)^{-\beta}\right]^{3}\right\} \\
S(x)=1-(1-\lambda)\left(1+\alpha x^{-\theta}\right)^{-\beta}-3 \lambda\left(1+\alpha x^{-\theta}\right)^{-2 \beta}+2 \lambda\left(1+\alpha x^{-\theta}\right)^{-3 \beta}
\end{gathered}
$$

With various choice of parametric values the Figure 3 illustrate the survival function pattern of cubic transmuted Dagum distribution is shown below:
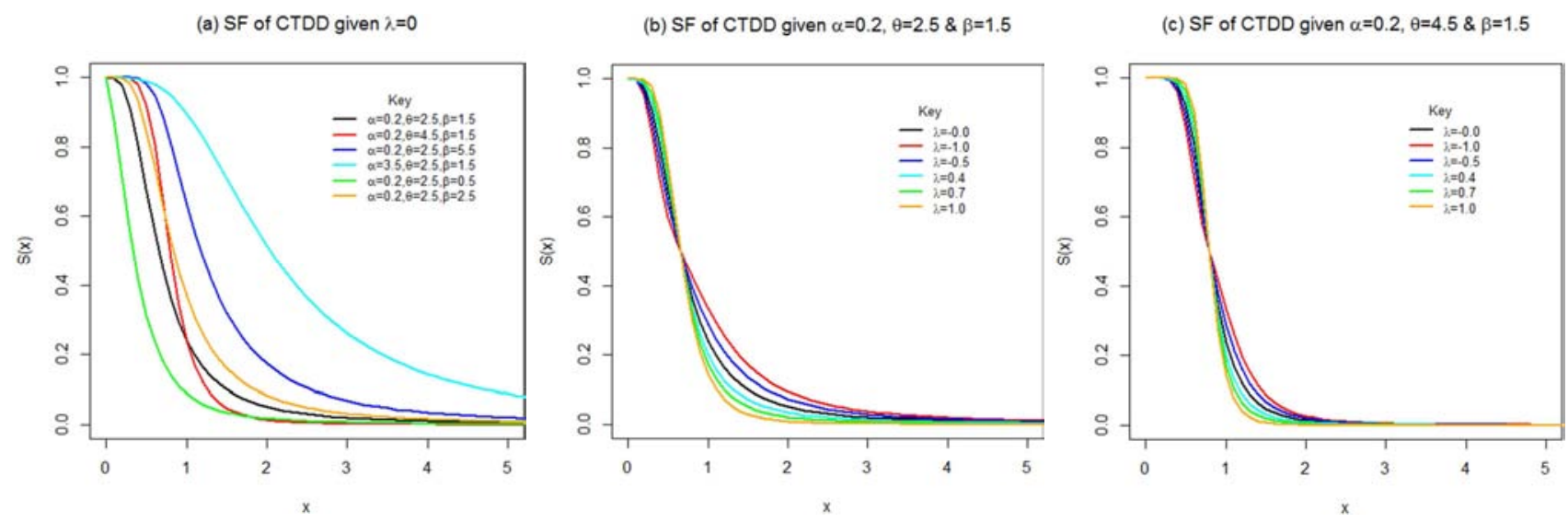

(d) $\mathrm{SF}$ of $\mathrm{CTDD}$ given $\alpha=0.2, \theta=2.5 \& \beta=5.5$

(e) $\mathrm{SF}$ of $\mathrm{CTDD}$ given $\alpha=3.5, \theta=2.5 \& \beta=1.5$

(f) $S F$ of CTDD given $\alpha=0.2, \theta=2.5 \& \beta=2.5$
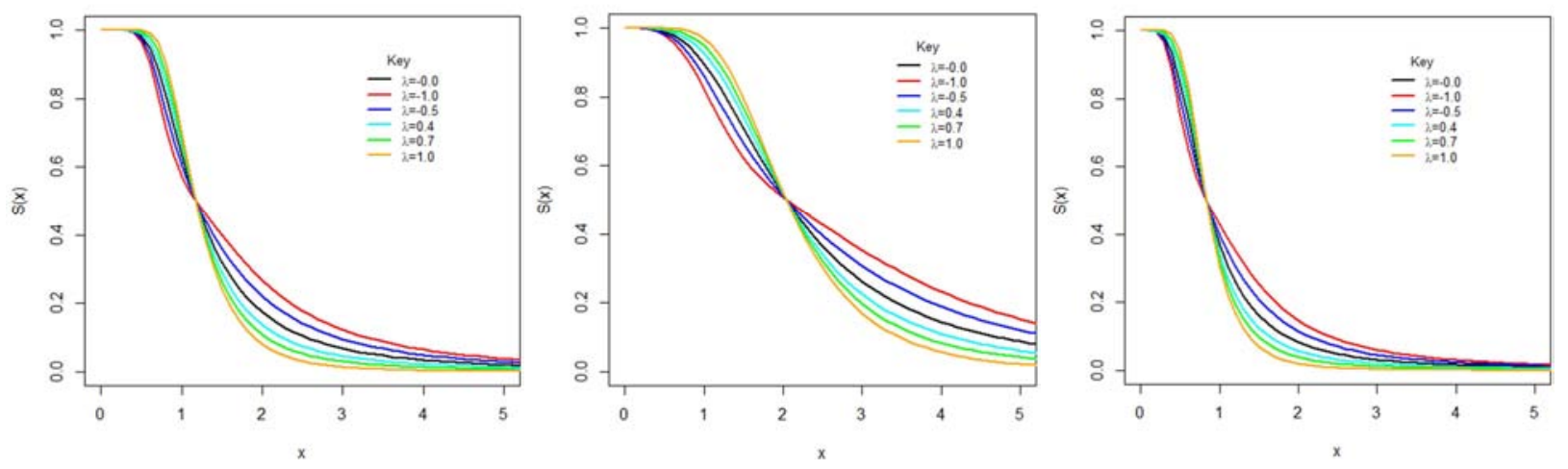

Figure 3. Survival Function of the CTDD for different values of the parameters.

\subsubsection{Hazard Function}

Hazard function is the failure function, and is the probability that a component will fail or die for an interval period of time. The hazard function is defined as

$h(x)=\frac{f(x)}{1-F(x)}=\frac{f(x)}{s(x)}$ where $F(x)$ and $\mathrm{f}(x)$ are the cdf and pdf of the proposed cubic transmuted Dagum distribution and is given as:

$$
\begin{gathered}
h(x)=\frac{\alpha \theta \beta x^{-\theta-1}\left\{\left(1+\alpha x^{-\theta}\right)^{-\beta-1}[1-\lambda+6 \lambda]\left(1+\alpha x^{-\theta}\right)^{-\beta}-6 \lambda\left[\left(1+\alpha x^{-\theta}\right)^{-\beta}\right]^{2}\right\}}{1-\left\{(1-\lambda)\left(1+\alpha x^{-\theta}\right)^{-\beta}+3 \lambda\left[\left(1+\alpha x^{-\theta}\right)^{-\beta}\right]^{2}-2 \lambda\left[\left(1+\alpha x^{-\theta}\right)^{-\beta}\right]^{3}\right\}} \\
h(x)=\frac{\alpha \theta \beta x^{-\theta-1}\left\{\left(1+\alpha x^{-\theta}\right)^{-\beta-1}[1-\lambda+6 \lambda]\left(1+\alpha x^{-\theta}\right)^{-\beta}-6 \lambda\left[\left(1+\alpha x^{-\theta}\right)^{-\beta}\right]^{2}\right\}}{1-(1-\lambda)\left(1+\alpha x^{-\theta}\right)^{-\beta}-3 \lambda\left(1+\alpha x^{-\theta}\right)^{-2 \beta}+2 \lambda\left(1+\alpha x^{-\theta}\right)^{-3 \beta}} \\
h(x)=\frac{\alpha \theta \beta x^{-\theta-1}\left\{1-\lambda+6 \lambda\left(1+\alpha x^{-\theta}\right)^{-\beta}-6 \lambda\left(1+\alpha x^{-\theta}\right)^{-2 \beta}\right\}}{\left(1+\alpha x^{-\theta}\right)\left\{1-\lambda+3 \lambda\left(1+\alpha x^{-\theta}\right)^{-\beta}-2 \lambda\left(1+\alpha x^{-\theta}\right)^{-2 \beta}\right\}}
\end{gathered}
$$

With various choice of parametric values the Figure 4 illustrate the hazard function pattern of cubic transmuted Dagum distribution is shown below: 

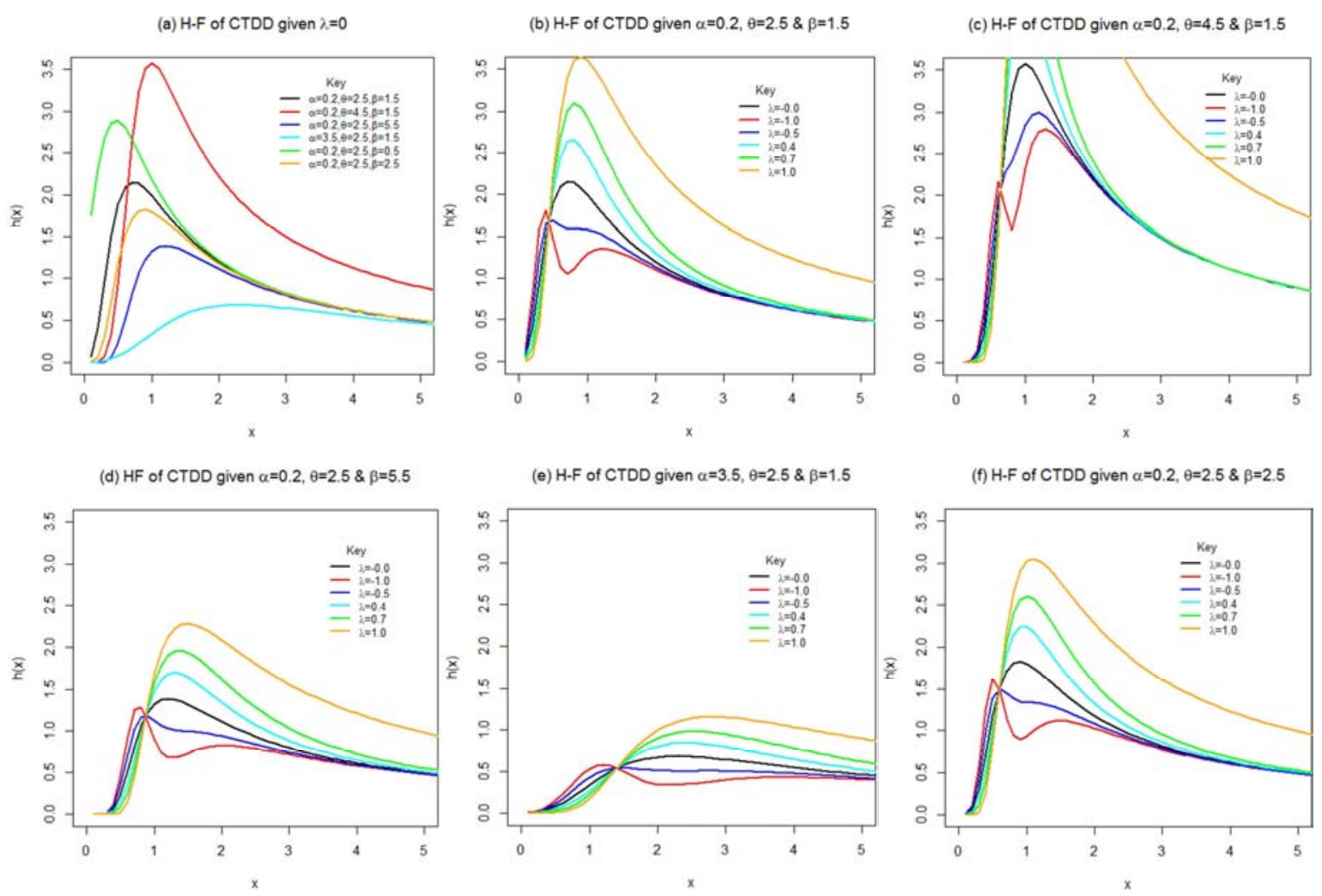

Figure 4. Hazard Function of the CTDD for different values of the parameters.

\subsubsection{Order Statistics}

In probability statistics the distribution of the extremes (smallest and / or largest), are the most important functions of a random variable. This is only the order statistics that help us to study the peaks of a data to understand the pattern of the extremes. Mathematically the order statistics is defined as, let $X_{(1)} \leq X_{(2)} \leq \cdots \leq X_{(n)}$ then the value $X_{(1)}, X_{(2)}, \ldots X_{(n)}$ are the order statistics of random variable. Suppose $X_{1}, X_{2}, \ldots X_{n}$ is a random sample from a distribution with probability density function $f(x)$, and let $X_{1: n}, X_{2: n}, \ldots X_{i: n}$ denote the corresponding order statistics obtained from this sample. The pdf, $f_{i: n}(x)$ of the $i^{t h}$ order statistic can be defined as:

$$
f_{i: n}(x)=\frac{n !}{(i-1) !(n-i) !} f(x) F(x)^{i-1}[1-F(x)]^{n-i}
$$

Where $f(x)$ and $\mathrm{F}(x)$ are the pdf and cdf of the proposed distribution respectively.

Using (5) and (6), the pdf of the $i^{\text {th }}$ order statistics $X_{i: n}$, can be expressed from (17) as

$$
\begin{gathered}
f_{i: n}(x)=\frac{n !}{(i-1) !(n-i) !} \sum_{k=0}^{n-i}(-1)\left(\begin{array}{c}
n-i \\
k
\end{array}\right)\left\{\alpha \theta \beta x^{-\theta-1}\left(1+\alpha x^{-\theta}\right)^{-\beta-1}\left[1-\lambda+6 \lambda\left(1+\alpha x^{-\theta}\right)^{-\beta}-6 \lambda\left(1+\alpha x^{-\theta}\right)^{-2 \beta}\right]\right\}\{(1- \\
\left.\lambda)\left[\left(1+\alpha x^{-\theta}\right)^{-\beta}\right]+3 \lambda\left[\left(1+\alpha x^{-\theta}\right)^{-\beta}\right]^{2}-2 \lambda\left[\left(1+\alpha x^{-\theta}\right)^{-\beta}\right]^{3}\right\}^{i+k-1}
\end{gathered}
$$

Hence, the pdf of the minimum order statistic $X_{(1)}$ and the maximum order statistic $X_{(n)}$ of the cubic transmuted Dagum distribution are given by

$$
\begin{gathered}
f_{1: n}(x)=\frac{n !}{(i-1) !(n-i) !} n \sum_{k=0}^{n-i}(-1)^{k}\left(\begin{array}{c}
n-i \\
k
\end{array}\right)\left\{\alpha \theta \beta x^{-\theta-1}\left(1+\alpha x^{-\theta}\right)^{-\beta-1}\left[1-\lambda+6 \lambda\left(1+\alpha x^{-\theta}\right)^{-\beta}-6 \lambda\left(1+\alpha x^{-\theta}\right)^{-2 \beta}\right]\right\}\{(1- \\
\left.\lambda)\left[\left(1+\alpha x^{-\theta}\right)^{-\beta}\right]+3 \lambda\left[\left(1+\alpha x^{-\theta}\right)^{-\beta}\right]^{2}-2 \lambda\left[\left(1+\alpha x^{-\theta}\right)^{-\beta}\right]^{3}\right\}^{k}
\end{gathered}
$$

And

$$
\begin{gathered}
f_{n: n}(x)=n\left\{\alpha \theta \beta x^{-\theta-1}\left(1+\alpha x^{-\theta}\right)^{-\beta-1}\left[1-\lambda+6 \lambda\left(1+\alpha x^{-\theta}\right)^{-\beta}-6 \lambda\left(1+\alpha x^{-\theta}\right)^{-2 \beta}\right]\right\}\left\{(1-\lambda)\left[\left(1+\alpha x^{-\theta}\right)^{-\beta}\right]+\right. \\
\left.3 \lambda\left[\left(1+\alpha x^{-\theta}\right)^{-\beta}\right]^{2}-2 \lambda\left[\left(1+\alpha x^{-\theta}\right)^{-\beta}\right]^{3}\right\}^{n-1}
\end{gathered}
$$

Respectively. 


\section{Application}

In this section, we provide a data analysis in order to assess the goodness of fit of the

Model as shown below.

Data set I: The first data set was obtained [14] corresponds to the time of successive failures of the air conditioning system of jet airplanes are:

$194,413,90,74,55,23,97,50,359,50,130,487,102,15,14,10,57,320,261,51,44,9,254,493,18,209,41,58,60,48$, $56,87,11,102,12,5,100,14,29,37,186,29,104,7,4,72,270,283,7,57,33,100,61,502,220,120,141,22,603,35,98$, $54,181,65,49,12,239,14,18,39,3,12,5,32,9,14,70,47,62,142,3,104,85,67,169,24,21,246,47,68,15,2,91,59$, $447,56,29,176,225,77,197,438,43,134,184,20,386,182,71,80,188,230,152,36,79,59,33,246,1,79,3,27,201,84$, $27,21,16,88,130,14,118,44,15,42,106,46,230,59,153,104,20,206,5,66,34,29,26,35,5,82,5,61,31,118,326,12$, $54,36,34,18,25,120,31,22,18,156,11,216,139,67,310,3,46,210,57,76,14,111,97,62,26,71,39,30,7,44,11,63$, $23,22,23,14,18,13,34,62,11,191,14,16,18,130,90,163,208,1,24,70,16,101,52,208,95$. The summary of dataset I is also provided in Table 1 as follows:

Table 1. Summary Statistics for the dataset I.

\begin{tabular}{|c|c|c|c|c|c|c|c|c|c|}
\hline $\mathbf{N}$ & Minimum & $Q_{1}$ & Median & $Q_{3}$ & Mean & Maximum & Variance & Skewness & Kurtosis \\
\hline 213 & 1.00 & 22.00 & 57.00 & 118.00 & 93.1409 & 603.00 & 11398.47 & 2.11185 & 4.92499 \\
\hline
\end{tabular}

Table 2. Maximum Likelihood Parameter Estimates for dataset I.

\begin{tabular}{lllll}
\hline Distribution & $\widehat{\boldsymbol{\alpha}}$ & $\widehat{\boldsymbol{\theta}}$ & $\widehat{\boldsymbol{\beta}}$ & $\hat{\boldsymbol{\lambda}}$ \\
\hline CTDD & 6.9182108 & 0.7627706 & 2.2957751 & 0.8781492 \\
TDD & 8.7601516 & 0.7522840 & 3.2381222 & 0.9153611 \\
DD & 9.2604712 & 0.9584467 & 3.4549300 & - \\
\hline
\end{tabular}

Table 3. The statistics $\ell, A I C, C A I C, B I C$ and HQIC for dataset $I$.

\begin{tabular}{lllllll}
\hline Distribution & $\hat{\boldsymbol{\ell}}$ & $\boldsymbol{A I C}$ & $\boldsymbol{C A I C}$ & $\boldsymbol{B I C}$ & HQIC \\
\hline CTDD & 1185.221 & 2378.442 & 2378.635 & 2391.887 & 2383.876 \\
TDD & 1185.479 & 2378.959 & 2379.151 & 2392.404 & 2384.392 \\
DD & 1193.004 & 2392.009 & 2392.124 & 2402.093 & 2396.084 & $2^{\text {st }}$ \\
\hline
\end{tabular}

Table 4. The $A^{*}, W^{*}, K$-S statistic and P-values for dataset $I$.

\begin{tabular}{lllll}
\hline Distribution & $\mathbf{A}^{*}$ & $\mathbf{W}^{*}$ & K-S & P-Value (K-S) \\
\hline CTDD & 1.474723 & 0.2216449 & 0.053947 & 0.5649 \\
TDD & 1.303243 & 0.1920248 & 0.064206 & 0.3437 \\
DD & 2.487181 & 0.3819815 & 0.075145 & $1^{\text {st }}$ \\
\hline
\end{tabular}

(a) Estimated Pdfs for Dataset I

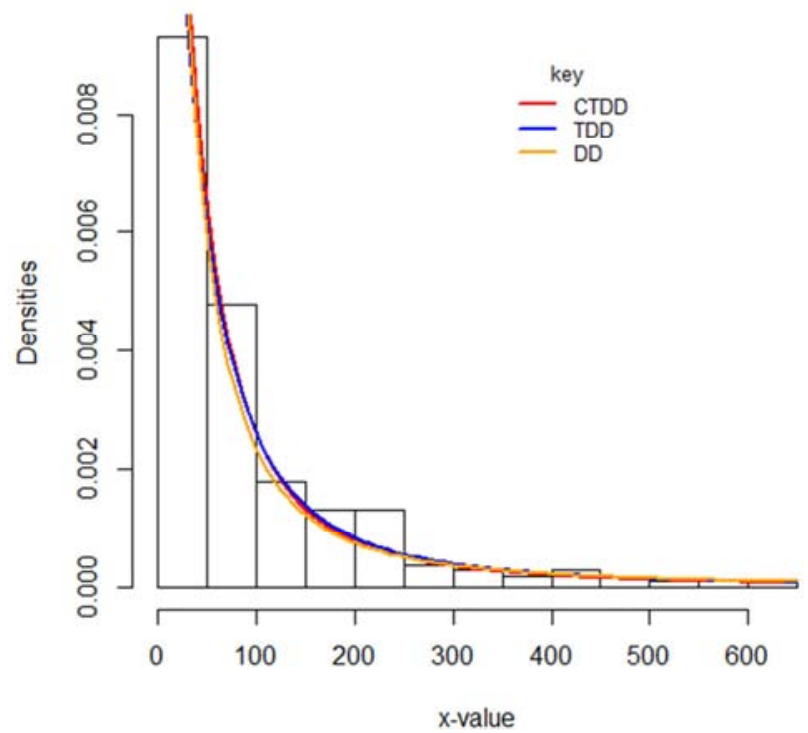

(b) Estimated Cdfs for Dataset I

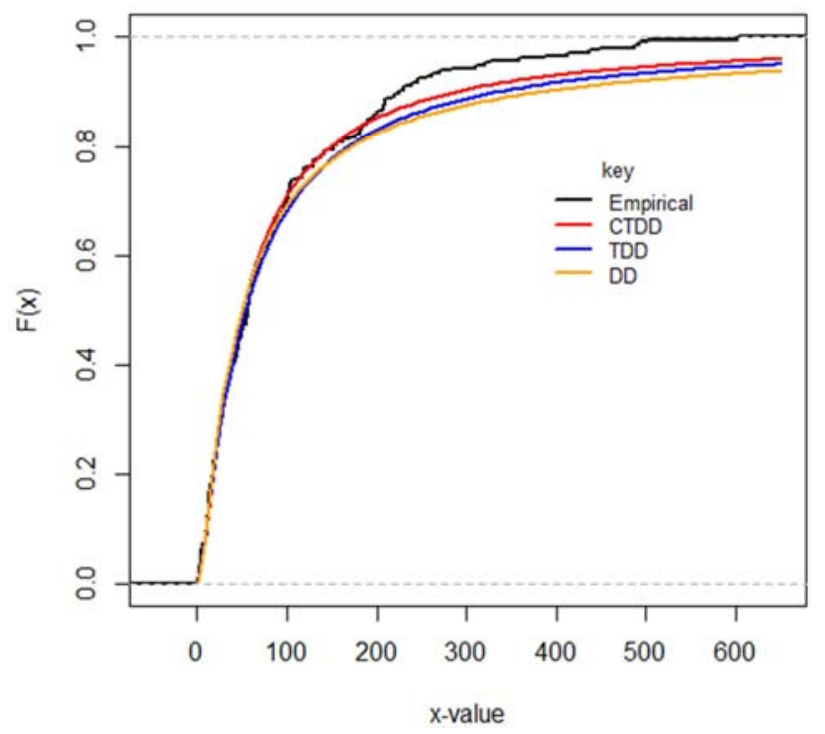

Figure 5. Histogram and plots of the estimated densities and cdfs of the CTDD and other fitted distributions to dataset I. 
(a) CTDD

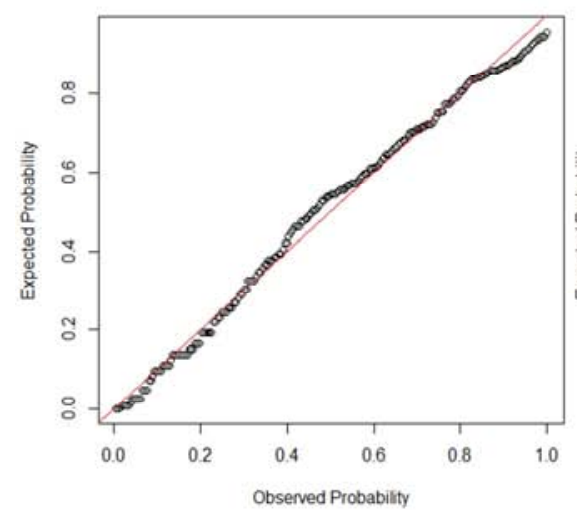

(b) TDD

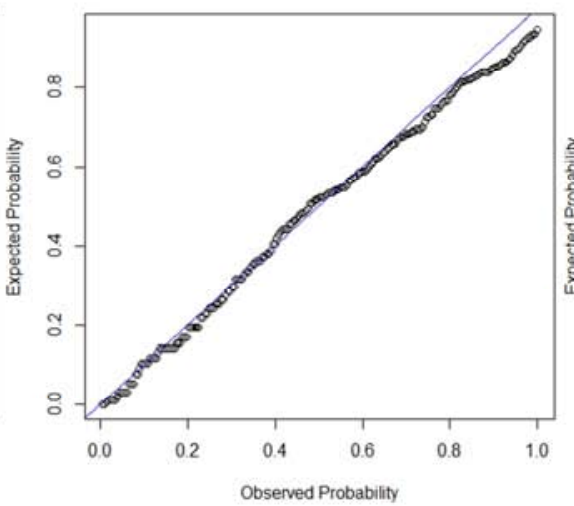

(c) DD

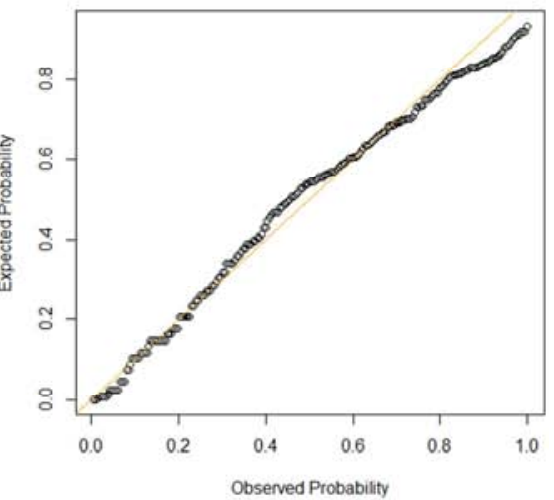

Figure 6. Probability plots for the fit of the CTDD and other fitted models based on dataset I.

Dataset II: This dataset represents 59 observations of the monthly actual taxes revenue in Egypt (in 1,000 million Egyptian pounds) between January 2006 and November 2010. The data has been previously used [15]. The descriptive statistics for this data are as follows:

(a) Estimated Pdfs for Dataset II

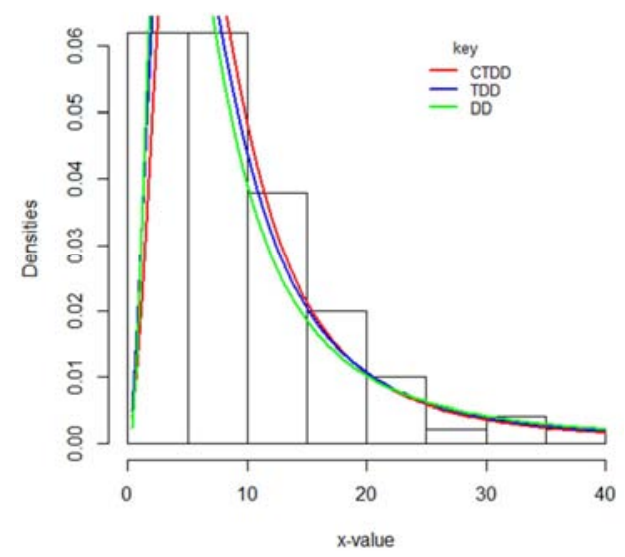

(b) Estimated Cdfs for Dataset II

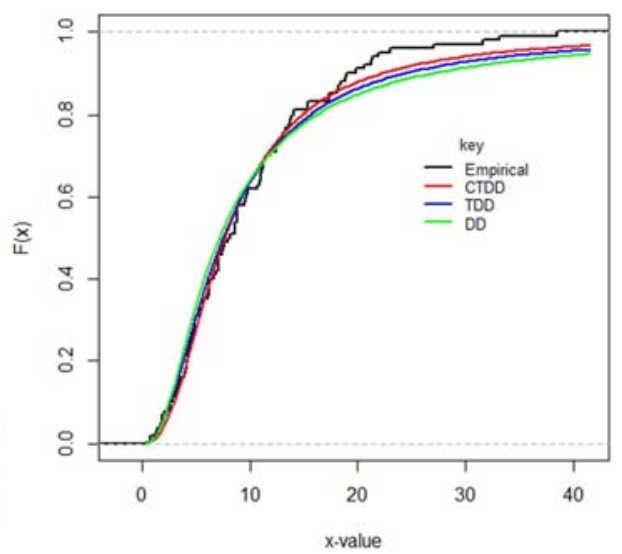

Figure 7. Histogram and plots of the estimated densities and cdfs of the CTDD and other fitted distributions to dataset II.

(a) CTDD

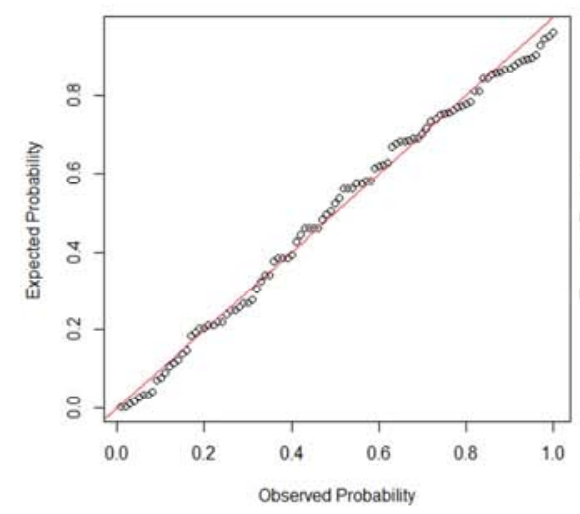

(b) TDD

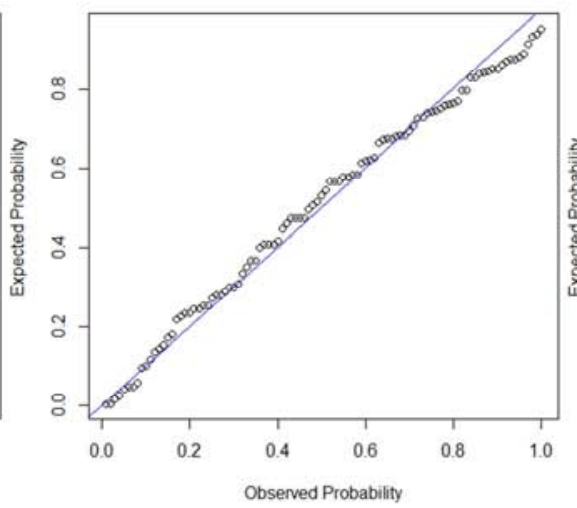

(c) DD

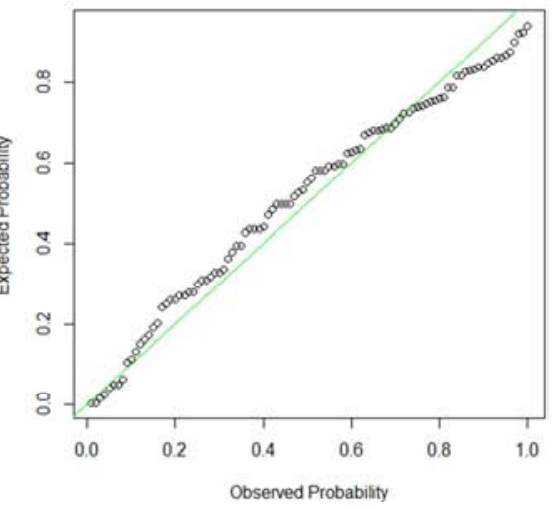

Figure 8. Probability plots for the fit of the CTDD and other fitted models based on dataset II.

\section{Conclusion}

In this article, we have introduced the cubic transmuted Dagum distribution. The distribution is flexible enough to capture the complexity of the data. We have derived expressions for the moments, generating functions, quantile function, random number generation, reliability function, order statistics. The maximum likelihood estimation of parameters for cubic transmuted Dagum distribution has also been discussed. We have conducted data analysis on two sets of Data and have applied the cubic transmuted Dagum distribution on the two real data sets. We have observed that the cubic transmuted Dagum distribution provides better fit 
than Dagum and transmuted Dagum distributions for the used data sets.

Table 5. Summary Statistics for the dataset II

\begin{tabular}{lllllllllll}
\hline Parameters & $\boldsymbol{N}$ & Minimum & $\boldsymbol{Q}_{\mathbf{1}}$ & Median & $\boldsymbol{Q}_{\mathbf{3}}$ & Mean & Maximum & Variance & Skewness & Kurtosis \\
\hline Values & 59 & 4.10 & 8.45 & 10.60 & 16.85 & 13.49 & 39.20 & 64.8266 & 1.6083 & 2.256 \\
\hline
\end{tabular}

Table 6. Maximum Likelihood Parameter Estimates for dataset II.

\begin{tabular}{lllll}
\hline Distribution & $\widehat{\boldsymbol{\alpha}}$ & $\widehat{\boldsymbol{\theta}}$ & $\hat{\boldsymbol{\beta}}$ & \\
\hline CTDD & 7.8353941 & 1.3914289 & 1.8250880 & 0.6961304 \\
TDD & 9.7243708 & 1.3532238 & 2.2196971 & 0.7483733 \\
DD & 5.636373 & 1.494778 & 2.655083 & - \\
\hline
\end{tabular}

Table 7. The statistics $\ell, A I C, C A I C, B I C$ and HQIC for dataset II

\begin{tabular}{lllllll}
\hline Distribution & $\hat{\boldsymbol{\ell}}$ & $\boldsymbol{A I C}$ & $\boldsymbol{C A I C}$ & $\boldsymbol{B I C}$ & $\boldsymbol{H Q I C}$ \\
\hline CTDD & 321.4249 & 650.8498 & 651.2709 & 661.2705 & 655.0673 \\
TDD & 322.3794 & 652.7588 & 653.1798 & 663.1795 & 656.9762 \\
DD & 325.4533 & 656.9067 & 657.1567 & 664.7222 & $1^{\text {st }}$ & 660.0698 \\
\hline
\end{tabular}

Table 8. The $A^{*}, W^{*}, K$-S statistic and P-values for dataset II.

\begin{tabular}{lllll}
\hline Distribution & $\mathbf{A}^{*}$ & $\mathbf{W}^{*}$ & K-S & P-Value (K-S) \\
\hline CTDD & 0.6371453 & 0.09166678 & 0.055895 & 0.9135 \\
TDD & 0.6749832 & 0.09511819 & 0.070874 & 0.6966 \\
DD & 1.112922 & 0.1615126 & 0.085802 & $1^{\text {st }}$ \\
\hline
\end{tabular}

\section{References}

[1] Dagum, C. (1983). Income distribution models. In: S. Kotz, N. L. Johnson, and C. Read (eds.): Encyclopedia of Statistical Sciences, Vol. 4. New York: John Wiley, pp. 27-34.

[2] Dagum, C. (1980). The generation and distribution of income, the Lorenz curve and the Gini ratio. In Silva, A. O, Cecilia, L. M \& Cordeiro, G. M (2017). The extended Dagum Distribution: Properties and Application. Journal of Data Science, 13, 53-73.

[3] Dagum, C. (1977). A new model of personal income distribution: Specification and estimation. Economie Appliquée, 30, 413-437. In Silva, A. O, Cecilia, L. M \& Cordeiro, G. M (2017). The Extended Dagum Distribution: Properties and Application. Journal of Data Science, 13 5373.

[4] Elbatal I and Aryal G (2015). Transmuted Dagum distribution with Applications. Chilean journal of Statistics 6 (2), 31-45.

[5] Granzotto, D. C. T., Louzada, F., and Balakrishnan, N. (2017). Cubic rank transmuted distributions: inferential issues and applications. Journal of Statistical Computation and Simulation, 87: 2760-2778, doi. 10.1080/00949655.2017.1344239.

[6] Shahzad M. N and Asghar Z (2016). Transmuted Dagum Distribution: A more flexible and broad Shaped hazard function model. Hacettepe Journal of Mathematics and Statistics. Vol 45 (1), 227-244.

[7] Fattorini, L. Lemmi, A. (1979) Proposta di un modello alternativo per lanalisi della distribuzione personale del reddito, Atti Giornate di Lavoro AIRO 28 (1), 89-117.
[8] Bordley, R. F., McDonald, J. B. and Mantrala, A. (1996) Something new, something old: Paramet- ric models for the size distribution of income, Journal of Income Distribution 6 (1), 91-103.

[9] Bandourian, R., McDonald, J. B. and Turley, R. S. (2003) A Comparison of Parametric Models of Income Distribution across Countries and Over Time, Estadistica 55, 135-152.

[10] Alwan, F. M., Baharum, A. and Hassan, G. S (2013). Reliability Measurement for Mixed Model Failures of 33/11 Kilovolt Electric Power Distribution Stations, PloS one 8 (8), $1-8$.

[11] Bird, J (2005) basic Engineering mathematics, $4^{\text {th }}$ ed. Elsevier Publihing: India Pg 108.

[12] Hyndman. R. J. and Fan, Y (1996). Sample quantiles in statistical packages. The American Statistician 50 (4), 361-365.

[13] Kalaa M. I and Adamu D (2013). Solving Quintin equation by Radicals. International journal of Research and Advancement in physical science. 3 (3): 75-77.

[14] Proschan, F. (1963). Theoretical Explanation of Observed Decreasing Failure Rate. Technometrics, 5, 375C383. https://doi.org/10.1080/00401706.1963.10490105.

[15] Owoloko, A \& Oguntunde, P \& Adejumo, A O. (2015). Performance rating of the transmuted exponential distribution: an analytical approach. SpringerPlus. 4. 10.1186/s40064-0151590-6.

[16] Rahman, M. M., Al-Zahrani, B., Shahbaz, S. H., and Shahbaz, M. Q. (2019b). Cubic Transmuted Uniform Distribution: An Alternative to Beta and Kumaraswamy Distributions. European Journal of Pure and Applied Mathematics, 12: 1106-1121. 